\title{
Allosteric Communication in the KIX Domain Proceeds through Dynamic Repacking of the Hydrophobic Core
}

\author{
Sven Brüschweiler, ${ }^{\dagger, \ddagger}{ }^{\ddagger}$ Robert Konrat, ${ }^{\ddagger}$ and Martin Tollinger*, ${ }^{\dagger}$ \\ ${ }^{\dagger}$ Institute of Organic Chemistry, Center for Molecular Biosciences Innsbruck (CMBI), University of Innsbruck, Innrain 80/82, \\ A-6020 Innsbruck, Austria \\ ${ }^{\ddagger}$ Structural and Computational Biology, Max F. Perutz Laboratories, Campus Vienna Biocenter 5, A-1030 Vienna, Austria
}

ABSTRACT: The KIX domain of the transcriptional coactivator CREB binding protein (CBP) co-operatively mediates interactions between transcription factors. Binding of the transcription factor mixed-lineage leukemia (MLL) induces the formation of a low-populated conformer of KIX that resembles the conformation of the KIX domain in the presence of a second transcription factor molecule. NMR spin relaxation studies have previously shown that allosteric coupling proceeds through a network of hydrophobic core residues that bridge the two binding sites. Here we describe high-resolution NMR solution structures of the binary complex of KIX with MLL and the ternary complex of KIX formed with MLL and

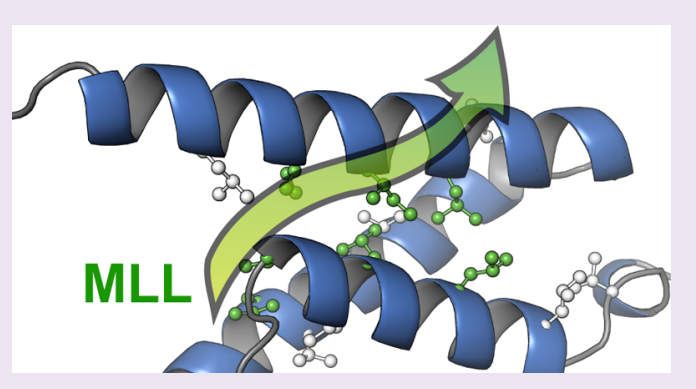
phosphorylated kinase inducible domain of CREB (pKID) as a second ligand. We show that binding of pKID to the binary complex of KIX with MLL is accompanied by a defined repacking of the allosteric network in the hydrophobic core of the protein. Rotamer populations derived from methyl group ${ }^{13} \mathrm{C}$ chemical shifts reveal a dynamic contribution to the repacking process that is not captured by the structural coordinates and exemplify the dynamic nature of allosteric communication in the KIX domain.

o-operativity plays a central role for the regulation of gene transcription. ${ }^{1-3}$ Transcription factors along with transcriptional coactivators assemble co-operatively on DNA promoter sequences, and transcription is stimulated by recruitment of RNA polymerase II. $^{4}$ These interactions are mediated by transcriptional coactivators, such as CREB binding protein (CBP), its paralog $\mathrm{p} 300$, or Mediator coactivator, which act as a scaffold for the recruitment of the transcriptional machinery. ${ }^{5,6}$ It has been observed in various studies that a combination of several DNA-bound transcription factors results in synergistic transcriptional response, ${ }^{1}$ and more specifically, several lines of evidence indicate that co-operative interactions between transcription factors and CBP are pivotal to promote synergism in transcriptional activation. ${ }^{3,7}$ CBP participates in the regulation of gene transcription by linking transcription factors with components of the basal transcriptional machinery. ${ }^{5}$ CBP is present at only low (and possibly limiting) concentrations in vivo, suggesting that competition of different transcription factors for CBP may be crucial for the regulation of gene transcription. ${ }^{8}$ The characterization of the biophysical mechanism by which co-operativity modulates the affinities of transcription factors for binding to $\mathrm{CBP}$ is a prerequisite for understanding how gene transcription is regulated.

CBP is a modular protein that contains a number of structured domains, as well as long stretches that are intrinsically disordered and represent linker regions or interaction motifs that fold only upon binding their biological targets. ${ }^{9}$ From a regulatory perspective, the KIX domain is of particular interest, since it is capable of binding two transcription factors simultaneously through two different binding sites, thereby directly mediating interactions between bound transcription factors. The three-dimensional structure of the KIX domain is composed of a bundle of three $\alpha$-helices and

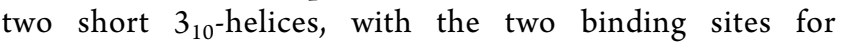
transcription factors, which are isolated from each other, being located at remote surfaces of the protein. ${ }^{10}$ Homologous KIX domains have been identified and characterized in p300 as well as in human and yeast Mediator coactivator subunits, and their structures were determined recently, revealing a high degree of functional and structural similarity. ${ }^{11,12}$

The KIX domain of CBP thus physically interlinks transcription factors by simultaneous binding through two interaction sites, and it does so in a co-operative and highly dynamic manner. ${ }^{13,14}$ In vitro, binding of the mixed-lineage leukemia (MLL) activation domain to KIX co-operatively enhances the interaction with the activation domain of the transcription factor c-Myb: KIX in complex with MLL displays $\sim 2$-fold higher affinity for c-Myb than the KIX domain alone. ${ }^{15}$ Likewise, positive co-operativity has been demonstrated for the interaction of the activation domain of pKID (the phosphorylated kinase inducible domain of CREB, which binds to KIX through the c-Myb interaction site) with KIX in complex with MLL. ${ }^{15}$ These co-operative effects provide a potential mechanism through which transcriptional activity might be modulated in the cell. Several observations relating to

Received: March 30, 2013

Accepted: May 7, 2013

Published: May 7, 2013 
communication between transcription factors mediated by CBP have indeed been described in the literature, suggesting transcriptional synergy between various transcription factors. $^{7,16}$

Using backbone and side chain nuclear magnetic resonance (NMR) relaxation dispersion techniques, we were able to monitor directly the conformational rearrangement process by which the KIX domain communicates information about the presence of a biological target at the MLL binding site to the allosterically regulated c-Myb/pKID binding site. ${ }^{17}$ NMR relaxation dispersion data revealed that binding the activation domain of MLL to KIX causes a redistribution of the relative populations of KIX conformers toward a state that adopts a conformation that is similar to that of the ternary complex. Titration experiments showed that this higher energy (excited) conformational substate of KIX.MLL, which is populated to $7 \%$ in the KIX.MLL binary complex, displays a higher affinity for c$\mathrm{Myb} / \mathrm{pKID}$ ligands than the $93 \%$ populated lower energy (ground) state of the protein complex. These results suggest that binding of $\mathrm{c}-\mathrm{Myb} / \mathrm{pKID}$ involves the selection of the higher energy conformer, whose structure is complementary to the ligand, from a pre-existing ensemble of conformations, reminiscent of the conformational selection mechanism of molecular recognition. ${ }^{18-20}$ In solution, KIX in complex with MLL is in an equilibrium between these two conformational substates within $\sim 3 \mathrm{~ms}$. As the higher affinity conformational substate is depleted from the equilibrium upon ligand binding, the equilibrium is recovered through the allosteric transition.

The exact structural basis of the allosteric transition is, however, unknown. The relaxation dispersion data indicate that a network of hydrophobic amino acids, which bridge the two binding sites of the KIX domain, constitutes the pathway through which allosteric information is communicated. The data suggest subtle conformational differences between lower and higher energy conformers, with chemical shift differences $\delta \omega$ between 0.4 and $1.0 \mathrm{ppm}$ found for the small subset of ${ }^{15} \mathrm{~N}$ and ${ }^{13} \mathrm{C}$ nuclei that form part of the allosteric network. This is contrasted by the remainder of the protein backbone, for which $\delta \omega$ values $<0.4 \mathrm{ppm}$ were detected, indicating that the structure of the three-helix scaffold is not affected by the transition between lower and higher energy conformers. Intriguingly, side chain methyl relaxation dispersion experiments performed on isoleucine $\delta 1$ methyl ${ }^{13} \mathrm{C}$ nuclei suggested a small but measurable conformational adaption of the part of the hydrophobic core that bridges the two binding sites. In addition, backbone amide ${ }^{1} \mathrm{H} /{ }^{2} \mathrm{H}$ exchange data showed that both (lower and higher energy) substates represent fully folded and solvent exchange protected conformers. ${ }^{17}$ Recent molecular dynamics (MD) simulations confirmed that the conformational ensemble of the KIX domain is shifted in response to the binding of $\mathrm{MLL}$, leading to a preorganized $\mathrm{c}-\mathrm{Myb} / \mathrm{pKID}$ binding site. $^{21}$

Here we report the NMR-derived high-resolution threedimensional solution structures of the binary complex of KIX formed with the activation domain of MLL and the ternary complex of KIX bound to MLL and pKID, based on NOEs and backbone chemical shifts and residual dipolar couplings. Validation of the side chain conformation of valine, leucine, and isoleucine residues using methyl ${ }^{13} \mathrm{C}$ chemical shift data establishes that these solution structures represent the highest populated rotamers of these residues in solution. However, the ${ }^{13} \mathrm{C}$ chemical shift data also reveal a defined redistribution of the rotameric states of residues in the hydrophobic core of the
KIX domain that occurs upon transition between the two complexes, which is not captured by the structural bundles. Our results thus facilitate a quantitative description of the dynamic structural adaption of the KIX domain in response to binding ligand molecules in terms of a redistribution of rotameric states of residues that form the hydrophobic core of the protein. In combination with pico- to nanosecond dynamic NMR experimental data, these results enable us to draw a comprehensive structural and dynamic picture of the molecular mechanism through which the KIX domain of CBP mediates co-operativity between transcription factors.

\section{MATERIALS AND METHODS}

Sample Preparation. Unlabeled, $10 \%{ }^{13} \mathrm{C}$-labeled, uniformly ${ }^{13} \mathrm{C}$-, ${ }^{15} \mathrm{~N}$-, or ${ }^{13} \mathrm{C}$, ${ }^{15} \mathrm{~N}$-labeled samples of the KIX domain (residues $586-$ 672) of human CBP were prepared by bacterial growth in standard LB and M9 minimal media and purified as described. ${ }^{22}$

Uniformly ${ }^{13} \mathrm{C},{ }^{15} \mathrm{~N}$-labeled MLL (residues 2840-2858; with C2841 substituted with alanine) was overexpressed in Escherichia coli as an $\mathrm{N}$ terminal fusion to a hexa-histidine tagged MBP. The cells were grown at $37{ }^{\circ} \mathrm{C}$ in $\mathrm{M} 9$ minimal media containing ${ }^{15} \mathrm{NH}_{4} \mathrm{Cl}$ and ${ }^{13} \mathrm{C}$-glucose as sole nitrogen and carbon sources in presence of kanamycin until $\mathrm{OD}_{600} \approx 0.6$, then the temperature was lowered to $28^{\circ} \mathrm{C}$, and after 30 min, protein synthesis was induced by adding isopropyl- $\beta$-Dthiogalactopyranoside (IPTG) to a final concentration of $0.8 \mathrm{mM}$. The cells were harvested $5 \mathrm{~h}$ after induction and resuspended in lysis buffer containing $20 \mathrm{mM}$ Tris- $\mathrm{HCl}(\mathrm{pH} 7.5), 250 \mathrm{mM} \mathrm{NaCl}, 10 \mathrm{mM}$ imidazole, and $5 \mathrm{mM} \beta$-mercaptoethanol. The cells were lysed using a French pressure cell and clarified by centrifugation. Subsequently, the supernatant was loaded onto a HisTrap FF crude (GE Healthcare) column. In the next step, the MBP-MLL fusion protein was applied to a Superdex 75 (GE Healthcare) equilibrated with cleavage buffer that contained $50 \mathrm{mM}$ Tris- $\mathrm{HCl}$ ( $\mathrm{pH} 7.5$ ), $0.5 \mathrm{mM}$ EDTA, and $2 \mathrm{mM}$ DTT. The His-tagged MBP was cleaved by incubation with TEV, and it was removed using nickel affinity chromatography. In the final step, MLL was purified to homogeneity by size exclusion chromatography using a Superdex 30 (GE Healthcare). Mass spectrometry was used to confirm the identity of MLL.

The human KID domain of CREB (residues 116-149) was prepared as a $\mathrm{N}$-terminal fusion to a hexa-histidine tagged thioredoxin. Uniformly ${ }^{13} \mathrm{C},{ }^{15} \mathrm{~N}$-labeled samples for NMR studies were prepared by growing E. coli cells in M9 minimal media containing ${ }^{15} \mathrm{NH}_{4} \mathrm{Cl}$ and ${ }^{13} \mathrm{C}$-glucose as sole nitrogen and carbon sources at $37{ }^{\circ} \mathrm{C}$. Protein overexpression was induced at $\mathrm{OD}_{600} \approx 0.8$ by adding IPTG to a final concentration of $0.7 \mathrm{mM}$. Cells were harvested after $5 \mathrm{~h}$ and trx-KID purification followed the same three-step procedure as established for MBP-MLL. Ser133 of KID was phosphorylated in vitro by incubation of $60 \mu \mathrm{M}$ purified KID with $0.05 \mu \mathrm{M}$ PKA catalytic subunit and $1 \mathrm{mM}$ ATP in a $25 \mathrm{mM}$ Tris- $\mathrm{HCl}(\mathrm{pH} 7)$ buffer containing $10 \mathrm{mM} \mathrm{MgCl}_{2}$ and $2 \mathrm{mM}$ DTT for $24 \mathrm{~h}$ at $30^{\circ} \mathrm{C}$. pKID was purified by size exclusion chromatography using a Superdex 30 (GE Healthcare). Mass spectrometry was used to confirm that pKID was fully phosphorylated.

NMR Spectroscopy. NMR spectra were recorded at $27{ }^{\circ} \mathrm{C}$ on Varian Inova 500 and $800 \mathrm{MHz}$ and Varian Direct Drive $600 \mathrm{MHz}$ spectrometers. Data were processed using NMRPipe ${ }^{23}$ and analyzed using CcpNmr. ${ }^{24} \mathrm{NMR}$ samples contained $1 \mathrm{mM}$ of labeled protein or peptide, unlabeled ligands in at least 2-fold excess, $50 \mathrm{mM}$ potassium phosphate buffer, pH 5.8, $25 \mathrm{mM} \mathrm{NaCl}$, and $1 \mathrm{mM} \mathrm{NaN}_{3}$ in $10 \%$ $\mathrm{D}_{2} \mathrm{O} / 90 \% \mathrm{H}_{2} \mathrm{O}$. Backbone and side chain ${ }^{1} \mathrm{H},{ }^{13} \mathrm{C}$, and ${ }^{15} \mathrm{~N}$ chemical shift assignments of KIX, pKID, and MLL were obtained using standard triple-resonance experiments: $\mathrm{HNCA} / \mathrm{HN}(\mathrm{CO}) \mathrm{CA}$, $\mathrm{HNCO} / \mathrm{HN}(\mathrm{CA}) \mathrm{CO}, \mathrm{CBCA}(\mathrm{CO}) \mathrm{NH} / \mathrm{HNCACB},{ }^{15} \mathrm{~N}$-edited TOCSY-HSQC, (H)CCONH-TOCSY, and HCCH-TOCSY. For the KIX domain in the complexes KIX·MLL and KIX·MLL·pKID, stereospecific assignment of the prochiral methyl groups of valine and leucine residues were obtained using the $10 \%$ fractional labeling method of Neri et al. ${ }^{25}$ Intramolecular distance restraints were obtained from 3D ${ }^{1} \mathrm{H}-{ }^{1} \mathrm{H}-\mathrm{NOESY}-{ }^{15} \mathrm{~N} /{ }^{13} \mathrm{C}-\mathrm{HSQC}$ experiments. 
Additional intramolecular distance restraints were obtained from 3D ${ }^{13} \mathrm{C},{ }^{13} \mathrm{C}$-methyl NOESY experiments. ${ }^{26}$ Intermolecular distance restraints were obtained from a $\omega_{1}{ }^{13} \mathrm{C}$-filtered simultaneous interand intramolecular three-dimensional ${ }^{1} \mathrm{H}-{ }^{1} \mathrm{H}$ NOESY $-{ }^{13} \mathrm{C}-\mathrm{HSQC}$ experiment. ${ }^{27}$ Residual dipolar couplings (RDCs) of KIX in both KIX.MLL and KIX.MLL·pKID and of pKID were measured on samples partially aligned using strain-induced alignment in a $4 \%$ polyacrylamide gel. ${ }^{28} \mathrm{HN}-\mathrm{N}$ RDCs $\left({ }^{1} \mathrm{D}^{1} \mathrm{H}_{1}{ }^{15} \mathrm{~N}\right)$ were measured using an in-phase/anti-phase (IPAP) ${ }^{1} \mathrm{H},{ }^{15} \mathrm{~N}-\mathrm{HSQC}$ experiment ${ }^{29}$ or a 3D besttype $\mathrm{HNCO}$ experiment. ${ }^{30}$

Heteronuclear ${ }^{1} \mathrm{H},{ }^{15} \mathrm{~N}$ NOE, rotating-frame longitudinal relaxation time $T_{1 \rho}$ and the longitudinal relaxation time $T_{1}$ were measured for KIX in the states KIX, KIX.MLL, KIX·pKID and KIX·MLL·pKID at $27^{\circ} \mathrm{C}$ and $800 \mathrm{MHz}{ }^{1} \mathrm{H}$ Larmor frequency. The heteronuclear ${ }^{1} \mathrm{H},{ }^{15} \mathrm{~N}$ NOE was obtained by recording, in an interleaved manner, one spectrum with a delay of $2 \mathrm{~s}$ followed by proton saturation for $3 \mathrm{~s}$ and another spectrum with a delay of $5 \mathrm{~s}$ without proton saturation. Relaxation delays of 10.9, 54.4, 108.9, 217.6, 326.4, 435.2, 598.4 and $707.2 \mathrm{~ms}$ were used for $T_{1}$ experiments, and delays of 10.0, 20.0, 30.0, $40.0,60.0,80.0$, and $100.0 \mathrm{~ms}$ were used for the $T_{1 \rho}$ measurements. The spherical diffusion tensor was determined by the method of Brüschweiler et al. ${ }^{31}$ using the program quadric_diffusion. ${ }^{32}$ The internal dynamics and overall tumbling were fit with the program FAST-ModelFree. $^{33}$

Structure Calculation and Refinement. Backbone dihedral angle restraints were set to $\phi=-60( \pm 10)^{\circ}$ and $\psi=-45( \pm 10)^{\circ}$ for residues that were predicted to be $\alpha$-helical based on ${ }^{13} \mathrm{C} \alpha,{ }^{13} \mathrm{C} \beta,{ }^{13} \mathrm{C}^{\prime}$, ${ }^{1} \mathrm{H} \alpha,{ }^{15} \mathrm{~N}$, and ${ }^{1} \mathrm{HN}$, using the software Talos plus. ${ }^{34}$ Distance restraints derived from the $3 \mathrm{D}{ }^{13} \mathrm{C},{ }^{13} \mathrm{C}$-methyl NOESY experiments were all set to an upper bound of $5.5 \AA$. All other intra- and intermolecular distance restraints were calibrated using the Aria2.3 program. ${ }^{35}$ An initial structural ensemble was used to determine the alignment tensor of KIX and pKID using the PALES software. ${ }^{36}$ The experimentally determined distance, dihedral angle, and dipolar coupling restraints were used in a torsion angle simulated annealing protocol using CNS1.2/Aria2.3 $3^{35,37}$ to solve the solution structure of the binary KIX·MLL complex and the ternary KIX.MLL.pKID complex. The final NMR ensembles were refined in an explicit water shell. ${ }^{38}$ The 20 lowest-energy solution structures (out of 100 calculated) were selected as a final representative ensemble of KIX·MLL and KIX·MLL·pKID.

\section{RESULTS}

Solution Structures of Binary and Ternary Complexes of KIX. NMR solution structures of the KIX domain of CBP (residues 586-672) were determined in the presence of the activation domain of the transcription factor MLL, as well as in the presence of the activation domains of both MLL and CREB (pKID), using triple-resonance-resolved NMR spectroscopic techniques, using backbone chemical shifts, backbone amide $\mathrm{NH}$ dipolar couplings, and NOEs as input restraints for the structure determination. Site-specific and stereospecific assignments of KIX isoleucine, valine, and leucine side chain methyl groups were obtained to enable a structural anlysis of the hydrophobic cores of the KIX domain in the binary and the ternary complexes. For KIX.MLL and KIX.MLL·pKID, all isoleucine side chain $\gamma 2$ and $\delta 1$ methyl resonances are resolved in two-dimensional $\left[{ }^{1} \mathrm{H},{ }^{13} \mathrm{C}\right]$ correlation experiments and could be assigned individually. In addition, we obtained stereospecific assignments of valine and leucine side chain methyl resonances for all 15 valine and leucine residues. Three-dimensional ${ }^{13} \mathrm{C},{ }^{13} \mathrm{C}$-methyl NOESY experiments ${ }^{26}$ were used to evaluate internuclear distances in the hydrophobic core of KIX in binary and ternary complexes KIX.MLL and KIX·MLL·pKID. Structural statistics are shown in Table 1. The KIX backbone structure of these complexes (Figure 1) is very similar to the
Table 1. NMR and Refinement Statistics for the KIX·MLL and KIX·MLL·pKID Complexes

\begin{tabular}{|c|c|c|}
\hline & KIX·MLL & KIX·MLL·pKID \\
\hline \multicolumn{3}{|c|}{ NMR Restraints } \\
\hline \multicolumn{3}{|l|}{ distance restraints } \\
\hline total NOE & 1016 & 934 \\
\hline intraresidue & $445 / 80$ & $309 / 74 / 92$ \\
\hline \multicolumn{3}{|l|}{ inter-residue } \\
\hline $\begin{array}{l}\text { sequential }(|i-j|= \\
1)\end{array}$ & $242 / 32$ & $180 / 32 / 55$ \\
\hline $\begin{array}{l}\text { medium-range }(\mid i- \\
j \mid \leq 4)\end{array}$ & $124 / 3$ & $77 / 3 / 12$ \\
\hline long-range $(|i-j| \geq$ & $70 / 0$ & $50 / 0 / 0$ \\
\hline intermolecular restraints & 20 & $23 / 27$ \\
\hline total dihedral restraints & 184 & 225 \\
\hline$\phi$ & $79 / 13$ & $79 / 13 / 20$ \\
\hline$\psi$ & $79 / 13$ & $79 / 13 / 21$ \\
\hline total ${ }^{1} \mathrm{D}_{\mathrm{H}-}^{1}{ }^{15} \mathrm{~N}$ RDCs & $80 / 0$ & $79 / 0 / 21$ \\
\hline \multicolumn{3}{|c|}{ Structure Statistics } \\
\hline \multicolumn{3}{|l|}{ violations (RMSD and SD) } \\
\hline distance restraints & $0.016 \pm 0.005$ & $0.073 \pm 0.02$ \\
\hline dihedral restraints & $1.2 \pm 0.1$ & $1.0 \pm 0.2$ \\
\hline${ }^{1} \mathrm{D}_{\mathrm{H}-}{ }^{15} \mathrm{~N}$ RDCs & $0.74 \pm 0.04$ & $0.90 \pm 0.10$ \\
\hline \multicolumn{3}{|l|}{ deviation from idealized geometry } \\
\hline bond lengths $(\AA)$ & $0.0041 \pm 0.0001$ & $0.0042 \pm 0.0002$ \\
\hline bond angles (deg) & $0.56 \pm 0.02$ & $0.59 \pm 0.02$ \\
\hline \multicolumn{3}{|l|}{ Ramachandran statistics } \\
\hline $\begin{array}{l}\text { residues in most favored } \\
\text { regions }\end{array}$ & $92.5 \%$ & $89.4 \%$ \\
\hline $\begin{array}{l}\text { residues in additional } \\
\text { allowed regions }\end{array}$ & $6.9 \%$ & $8.9 \%$ \\
\hline $\begin{array}{l}\text { residues in generously } \\
\text { allowed regions }\end{array}$ & $0.5 \%$ & $1.5 \%$ \\
\hline $\begin{array}{l}\text { residues in disallowed } \\
\text { regions }\end{array}$ & $0.1 \%$ & $0.3 \%$ \\
\hline \multicolumn{3}{|c|}{ Average Pairwise rms Deviation (A) } \\
\hline heavy atoms $\left(2^{\circ}\right.$ struct $)$ & $1.62 \pm 0.23$ & $1.82 \pm 0.25$ \\
\hline backbone atoms $\left(2^{\circ}\right.$ struct $)$ & $0.78 \pm 0.17$ & $0.92 \pm 0.22$ \\
\hline heavy atoms (all residues) & $2.71 \pm 0.59$ & $2.94 \pm 0.86$ \\
\hline backbone (all residues) & $2.18 \pm 0.64$ & $2.47 \pm 0.95$ \\
\hline
\end{tabular}

NMR structures of binary KIX complexes with $\mathrm{pKID},{ }^{39} \mathrm{c}$ $\mathrm{Myb}^{40}{ }^{4}$ and the PCET motif from HEB, ${ }^{41}$ as well as the ternary KIX complex formed with the MLL and c-Myb activation domains. ${ }^{10}$ The central scaffold of the KIX domain is formed by a bundle of three $\alpha$-helices (residues 597-611, $\alpha 1$; residues $623-642, \alpha 2$; residues $646-669, \alpha 3)$ together with two short

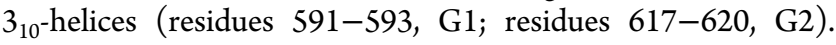
The three helices $\alpha 1-\alpha 3$ pack together in an antiparallel fashion to form an extended hydrophobic core, which is capped by the first $3_{10}$-helix G1 on one side, while the loop L12, which encompasses the $33_{10}$-helix G2 and connects helices $\alpha 1$ and $\alpha 2$, partly caps the other side of the hydrophobic core. ${ }^{10}$

In the binary complex KIX.MLL, as well as in ternary KIX·MLL·pKID, residues 2847-2855 of MLL form an amphipathic helix that binds to the hydrophobic groove on the surface of the KIX domain at the C-terminus of helix $\alpha 1$, similar to the ternary complex formed by KIX with the activation domains of MLL and c-Myb. ${ }^{10}$ Through the insertion of MLL into the hydrophobic groove, several hydrophobic residues (I2849, M2850, F2852, V2853, and L2854) of MLL are in the position to form hydrophobic contacts with a number of KIX residues (I611, F612, A619, R624 (aliphatic region), 

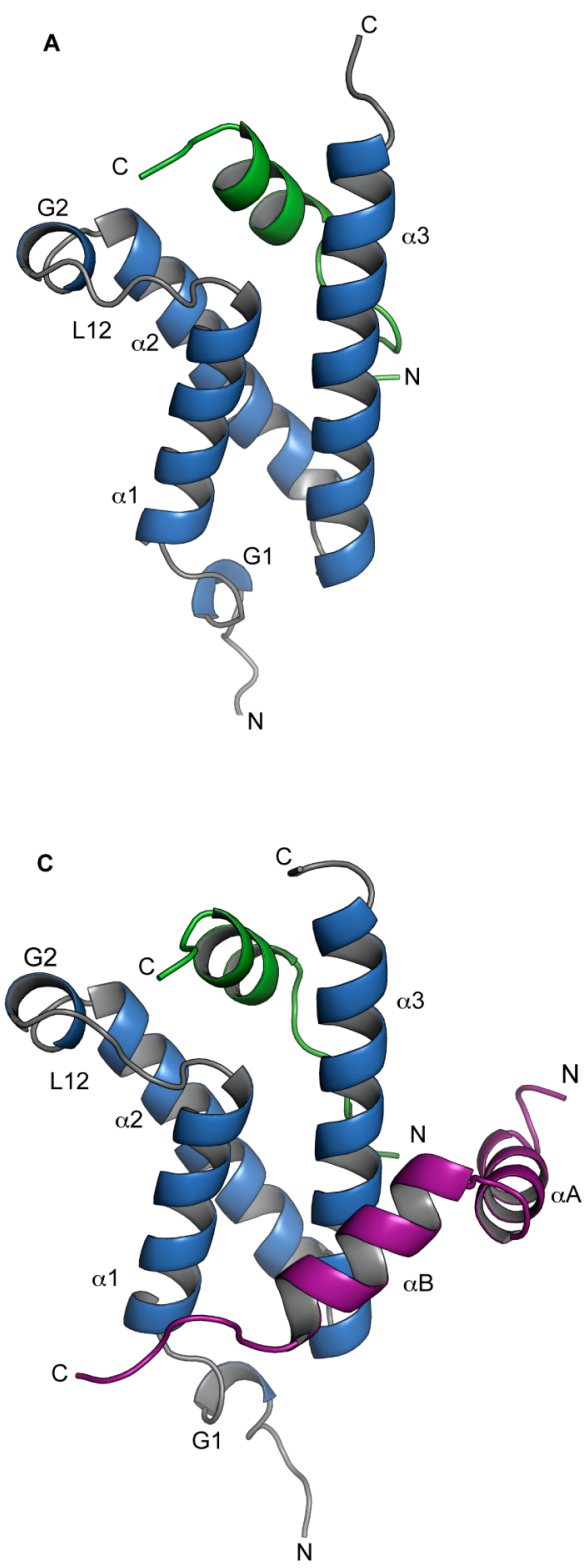

B

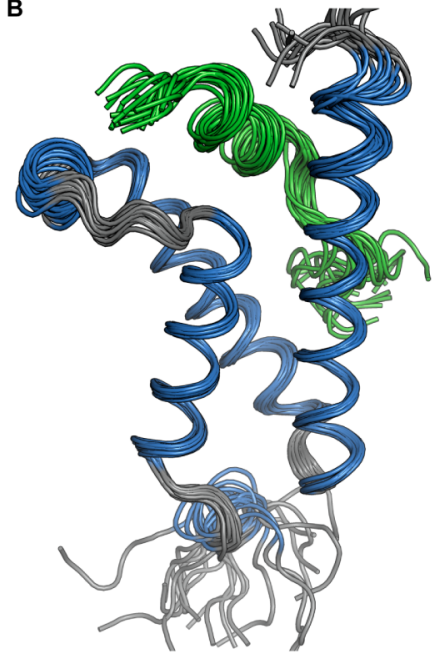

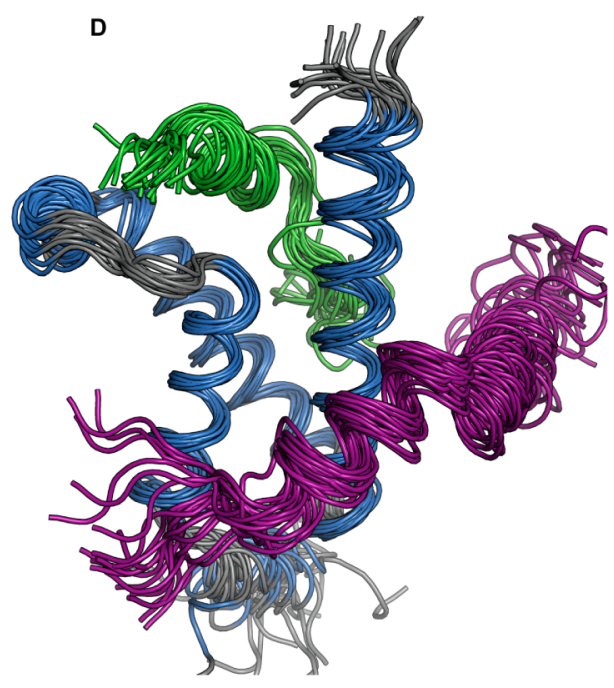

Figure 1. NMR solution structures of binary and ternary complexes of KIX. (A) Lowest energy structure of KIX in complex with MLL (residues 2840-2858); helices are displayed as ribbons. Secondary structure elements are labeled as according to Radhakrishnan et al. ${ }^{39}$ and color-coded (KIX, gray; helices, blue; MLL, green). (B) NMR ensemble of 20 representative structures of KIX.MLL (PDB ID 2LXS). (C) Lowest energy structure of KIX in complex with MLL (residues 2840-2858) and pKID (residues 116-149). Secondary structure elements are color-coded as in panel a (with pKID shown in magenta). (D) NMR ensemble of 20 representative structures of KIX·MLL·pKID (PDB ID 2LXT). All figures were generated using PyMOL. $^{64}$

L628, Y631, I660, and L664), which are part of the hydrophobic groove. In addition, the NMR structures indicate electrostatic interactions between the side chain of N2856 of MLL and two KIX side chains, T614 and D616. The structures of KIX.MLL and KIX.MLL·pKID complexes show no significant difference with respect to the binding mode of the MLL ligand peptide. Comparison of the KIX.MLL and KIX.MLL.pKID complexes with the binary complex between KIX and PCET reveals a slight difference in the orientation of the bound ligand peptide PCET versus MLL. ${ }^{41}$ This is reflected in a lower number of intermolecular contacts between KIX and PCET compared with MLL and has been related to the relatively low affinities of various PCET peptides for KIX. ${ }^{41}$ Of note, our NOE data do not indicate binding of the MLL peptide to additional binding surfaces on the KIX domain. ${ }^{42}$
In the ternary complex KIX.MLL·pKID, the pKID peptide binds into a shallow hydrophobic pocket on the surface of the KIX domain that is formed by side chains of amino acid residues in helices $\alpha 1$ ( $\mathrm{N}$-terminal part) and $\alpha 3$ (central part). Bound pKID forms two almost perpendicular helices $\alpha \mathrm{A}$ and $\alpha \mathrm{B}$, as observed previously for the binary complex of KIX formed with pKID. ${ }^{39}$ The side chains of helix $\alpha \mathrm{B}$ residues I137, L138, and L141 make close hydrophobic contacts with the pKID binding pocket formed by KIX helix $\alpha 3$ residues Y650, A654, I657, and Y658, while the side chain of pKID residue A145 loosely packs against the N-terminus of KIX helix $\alpha 1$ (L599 and L603). For the $\alpha$ A helix of pKID intermolecular NOEs were only found for residues I127 and L128, which make van der Waals contacts with Y658. In addition, the hydroxyl group of Y658 forms a hydrogen bond with the phosphate 

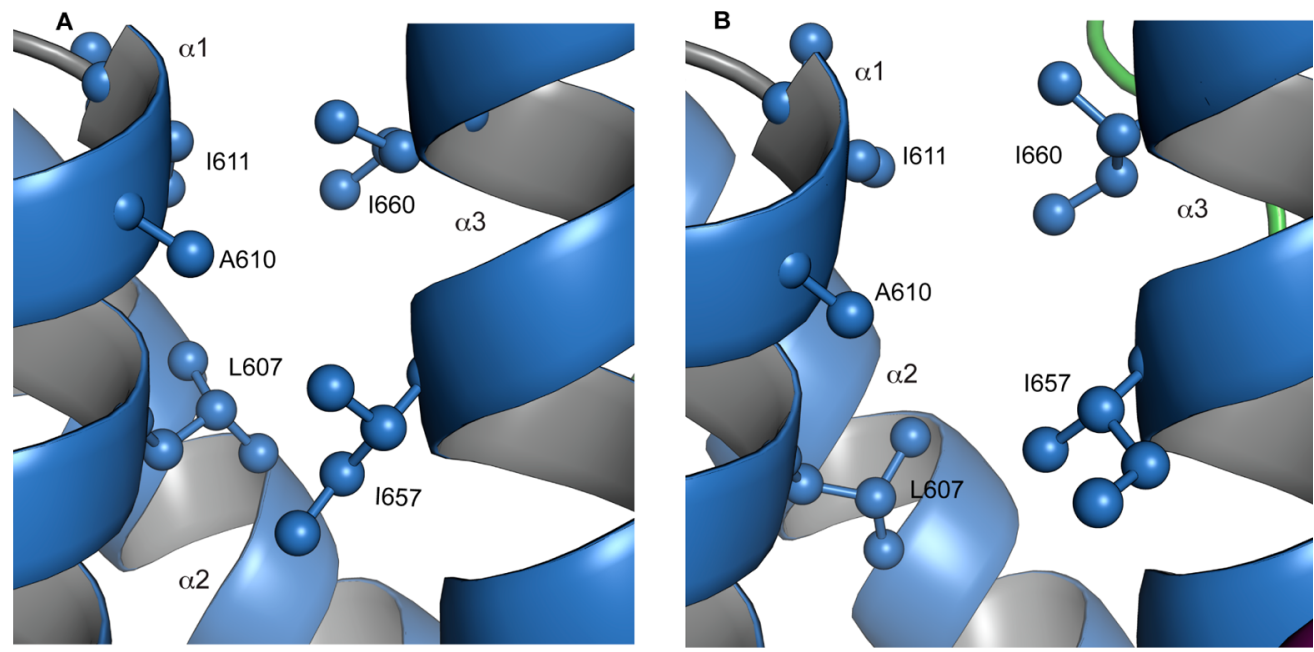

Figure 2. Close-up view of the hydrophobic core residues L607, A610, I611, I657, and I660 in (A) KIX·MLL and (B) KIX·MLL·pKID.

Table 2. Comparison of Side Chain Rotameric Distributions ( $\chi 2$ in Leucine and Isoleucine, $\chi 1$ in Valine) as Derived from Methyl ${ }^{13} \mathrm{C}$ Chemical Shifts ${ }^{a}$ with the NMR Solution Structures of KIX·MLL (2LXS) and KIX·MLL·pKID (2LXT)

\begin{tabular}{|c|c|c|c|c|c|}
\hline \multicolumn{3}{|c|}{ KIX·MLL } & \multicolumn{2}{|c|}{ KIX·MLL·pKID } & \multirow[b]{2}{*}{ change of $\chi^{2} \mathrm{t} / \mathrm{g}+(\%)$} \\
\hline & $\chi^{2} \mathrm{t} / \mathrm{g}+(\%)$ from ${ }^{13} \mathrm{C}$ shifts & $\chi 2$ rotamer in $2 \mathrm{LXS}$ & $\chi^{2} \mathrm{t} / \mathrm{g}+(\%)$ from ${ }^{13} \mathrm{C}$ shifts & $\chi^{2}$ rotamer in $2 \mathrm{LXT}$ & \\
\hline L599 & $33: 67$ & $g+$ & $32: 68$ & $g+$ & 1 \\
\hline L603 & 81:19 & $\mathrm{t}$ & $73: 27$ & $\mathrm{t}$ & 8 \\
\hline L607 & $57: 43$ & $\mathrm{t}$ & $62: 38$ & $\mathrm{t}$ & 5 \\
\hline L620 & $71: 29$ & $g-$ & $71: 29$ & $\mathrm{t}$ & 0 \\
\hline L628 & $43: 57$ & $\mathrm{~g}+$ & 29:71 & $\mathrm{g}+$ & 14 \\
\hline L652 & $88: 12$ & $\mathrm{t}$ & $87: 13$ & $\mathrm{t}$ & 1 \\
\hline L653 & $59: 41$ & $\mathrm{t}$ & $67: 33$ & $\mathrm{t}$ & 8 \\
\hline L664 & $80: 20$ & $\mathrm{t}$ & 80:20 & $\mathrm{t}$ & 0 \\
\hline L672 & $69: 31$ & $\mathrm{t}$ & $69: 31$ & $\mathrm{~g}+/ \mathrm{t} / \mathrm{g}-$ & 0 \\
\hline & $\chi^{2} \mathrm{t} / \mathrm{g}-(\%)$ from ${ }^{13} \mathrm{C}$ shifts & $\chi 2$ rotamer in $2 \mathrm{LXS}$ & $\chi^{2} \mathrm{t} / \mathrm{g}-(\%)$ from ${ }^{13} \mathrm{C}$ shifts & $\chi^{2}$ rotamer in $2 \mathrm{LXT}$ & change of $\chi^{2} \mathrm{t} / \mathrm{g}-(\%)$ \\
\hline I611 & $87: 13$ & $\mathrm{t}$ & $83: 17$ & $\mathrm{t}$ & 4 \\
\hline I657 & $74: 26$ & $\mathrm{t}$ & $67: 33$ & $\mathrm{t}$ & 7 \\
\hline $\mathrm{I} 660$ & $89: 11$ & $\mathrm{t}$ & $93: 7$ & $\mathrm{t}$ & 4 \\
\hline & $\chi 1 \mathrm{t} / \mathrm{g}+/ \mathrm{g}-(\%)$ from ${ }^{13} \mathrm{C}$ shifts & $\chi 1$ rotamer in $2 \mathrm{LXS}$ & $\chi 1 \mathrm{t} / \mathrm{g}+/ \mathrm{g}-(\%)$ from ${ }^{13} \mathrm{C}$ shifts & $\chi 1$ rotamer in $2 \mathrm{LXT}$ & change of $\chi 1 \mathrm{t} / \mathrm{g}+/ \mathrm{g}-(\%)$ \\
\hline V587 & $70: 9: 21$ & $\mathrm{t} / \mathrm{g}-$ & $70: 9: 21$ & $\mathrm{~g}+/ \mathrm{t} / \mathrm{g}-$ & 0 \\
\hline V595 & 90:0:10 & $\mathrm{t}$ & 89:0:11 & $\mathrm{t}$ & 1 \\
\hline V604 & 87:13:0 & $\mathrm{t}$ & $87: 13: 0$ & $t$ & 0 \\
\hline V608 & $73: 27: 0$ & $\mathrm{t}$ & $75: 25: 0$ & $\mathrm{t}$ & 2 \\
\hline V629 & 90:10:0 & $\mathrm{t}$ & 90:10:0 & $\mathrm{t}$ & 0 \\
\hline V635 & $98: 0: 2$ & $\mathrm{t}$ & 99:0:1 & $\mathrm{t}$ & 1 \\
\hline
\end{tabular}

$a_{\mathrm{t}}$ trans, $\mathrm{g}-$, gauche-; g+, gauche+; populations are given in percent $(\%)$, and the most populated rotameric state is printed in bold face.

group of pS133 of $\mathrm{pKID} .{ }^{39}$ Importantly, there are no direct contacts between the bound activation domains of MLL and pKID through which co-operativity between transcription factors could be mediated.

Structural Comparison. The pairwise rmsd between backbone atoms of the well-structured parts of the KIX domain (residues 589-669) in KIX.MLL and KIX.MLL·pKID is 1.07, which is comparable to the rms of the structural bundles of the two complexes ( 0.61 and 0.87 , respectively). This is consistent with the observation of only small chemical shift differences of backbone $\left({ }^{15} \mathrm{~N},{ }^{1} \mathrm{HN},{ }^{13} \mathrm{C}^{\alpha}\right)$ resonances between the binary and ternary complexes. ${ }^{17}$ It is therefore evident from the NMR structural analysis of the two complexes that the KIX protein backbone is not significantly affected by binding pKID. In addition, the methyl group NOE patterns are generally fairly similar for the binary and ternary complexes, consistent with well-defined and tightly packed hydrophobic cores. However, a number of significant differences between the NOE patterns of KIX.MLL and KIX.MLL·pKID indicate that the binding of pKID to binary KIX.MLL is accompanied by a small but measurable rearrangement of the hydrophobic core: for example, while relatively strong NOEs are observed, in the binary complex KIX.MLL, between the $\delta 1$ methyl group of I660 and both methyl groups of L607, these NOEs are absent in the ternary complex (along with their symmetry-equivalent NOEs in the three-dimensional experiments). Likewise, the NOE signal intensity between the $\delta 1$ methyl group of I657 and the backbone amide proton indicates a change in orientation of the I657 side chain upon binding pKID.

In Figure 2, the hydrophobic cores of KIX.MLL and KIX.MLL·pKID complexes are compared. In the binary complex KIX.MLL, the $\delta 1$ methyl group of 1660 is packed to the $\gamma 1$ methylene group of I611 and the methyl group of A610. Binding of $\mathrm{pKID}$, however, is accompanied by a change of the 
I660 side chain $\chi 1$ torsion angle, leading to an increase of the distance between the $\delta 1$ methyl group of I660 and the side chain methyls of residues I611, A610, and L607. Concomitantly, upon transition from binary KIX.MLL to ternary KIX.MLL.pKID, the side chain $\chi 1$ torsion angle of isoleucine I657 is rotated from gauche- to gauche+, thereby linking the conformational adaption of the hydrophobic core to the c$\mathrm{Myb} / \mathrm{pKID}$ binding surface of the KIX domain. In KIX.MLL·pKID, the $\delta 1$ methyl group of I657 forms close hydrophobic contacts with the side chains of I137 and L141 of the ligand peptide pKID.

Structural Validation Using ${ }^{13} \mathrm{C}$ Chemical Shifts. We used methyl carbon ${ }^{13} \mathrm{C}$ chemical shifts to validate the NOEbased hydrophobic packing of the KIX domain in the two complexes. Isoleucine $\left(\chi_{2}\right)$, leucine $\left(\chi^{2}\right)$, and valine $\left(\chi_{1}\right)$ rotameric distributions were determined from ${ }^{13} \mathrm{C}$ chemical shifts using quantitative expressions that are available for these residues. $^{43-45} \mathrm{~A}$ comparison of the chemical shift based populations of $\chi^{2}$ and $\chi 1$ rotamers with the dihedral angles of the NMR solution structures of binary KIX.MLL and ternary KIX.MLL.pKID is shown in Table 2, indicating a clear correspondence between the rotameric states observed in the three-dimensional structures and the ${ }^{13} \mathrm{C}$ chemical shift data in both complexes. For example, only for two (out of nine) leucine residues, L599 and L628, the $\chi 2$ gauche+ conformer is present in both complexes, while all other leucine residues populate the $\chi^{2}$ trans conformer. This is in very good agreement with the ${ }^{13} \mathrm{C}$ chemical shift data, from which $\chi^{2}$ gauche+ populations exceeding $50 \%$ are only predicted for L599 and L628 (with the exception of the highly dynamic Cterminal L672, for which a distribution of $\chi 2$ rotamers is found in ternary KIX.MLL·pKID, and L620, which populates the unusual gauche- conformer in binary KIX.MLL). Likewise, all three isoleucine residues are found to populate the $\chi^{2}$ trans conformer in our structures in both complexes, and all five well structured valine residues are found in $\chi 1$ trans conformation (only for the highly dynamic N-terminal V587 a distribution of $\chi 1$ rotamers is found in both complexes), consistent with their methyl group ${ }^{13} \mathrm{C}$ chemical shifts. Taken together, these data clearly show that the conformation in the solution structures of both complexes (binary KIX.MLL and ternary KIX.MLL.pKID) represents the most populated side chain rotameric states of isoleucine, leucine, and valine residues in all cases.

Pico- to Nanosecond Time Scale Dynamics of KIX and KIX Complexes. To characterize the dependence of the conformational flexibility of the KIX domain on the presence of bound ligands, we determined backbone amide order parameters, which are sensitive reporters of pico- to nanosecond time scale dynamics, ${ }^{46}$ for four different (complexed) states of the KIX domain: unliganded KIX, binary KIX.MLL, binary KIX.pKID, and ternary KIX·MLL·pKID (Figure 3 ). In binary and ternary complexes of KIX, the three-helix scaffold of the protein is well-ordered in solution, with backbone order parameters of ca. 0.9 for helices $\alpha 1-\alpha 3$. Order parameters of the unliganded KIX domain are measurably lower (the average of all order parameters in helices $\alpha 1-\alpha 3, S_{\text {avg }}^{2}$, is 0.85 ), suggesting a higher level of flexibility on the pico- to nanosecond time scale. Significantly lower than average order parameters are also found for the N-termini (including the $3_{10}$ helix G1) and the C-termini of the protein in all four states, consistent with the dynamic fraying of these segments of the protein that is observed in the structural bundles. In addition, the order parameters of the backbone amides in helix $\alpha 3$

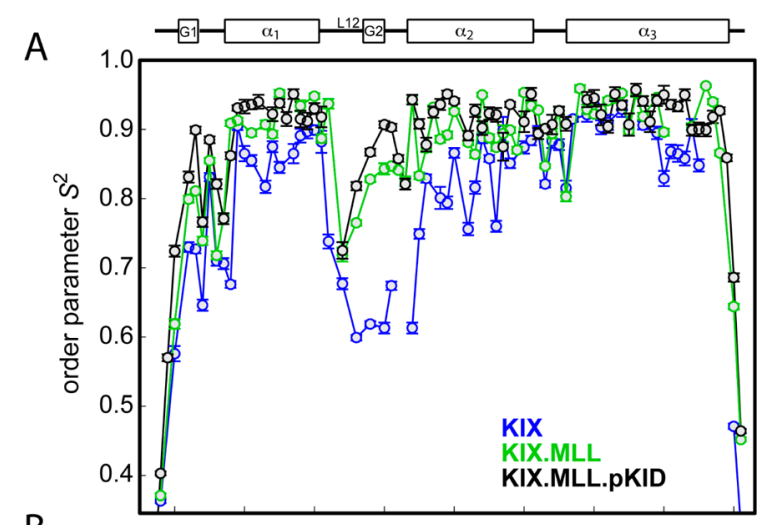

B

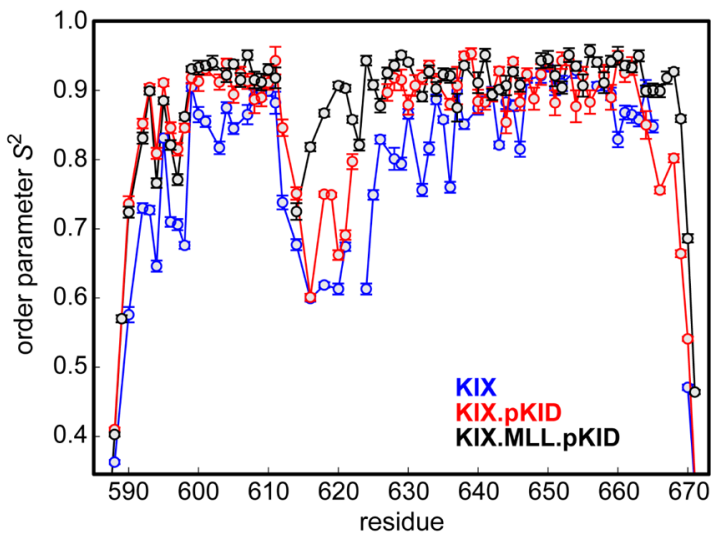

Figure 3. Comparison of backbone amide ${ }^{15} \mathrm{~N}$ order parameters $S^{2}$ in (A) KIX (blue), binary KIX·MLL (green), and ternary KIX.MLL·pKID (black) and (B) KIX (blue), binary KIX·pKID (red), and ternary KIX-MLL·pKID (black). Error bars are shown and lines between data points are drawn except in cases where $S^{2}$ could not be determined due to resonance overlap for two or more consecutive backbone amides.

suggest that the C-terminal half of this helix is more rigid in complexes containing MLL compared with unliganded KIX and KIX-pKID. This observation agrees with the low degree of dynamic fraying that is observed for the C-terminal half of helix $\alpha 3$ in the structural bundles of KIX.MLL and KIX.MLL·pKID. ${ }^{10}$

The NMR relaxation data also indicate significant pico- to nanosecond time scale dynamics in the loop region L12, along with a clear difference between different complexes of KIX. For this part of the KIX backbone, average order parameters vary in a stepwise manner upon binding ligands, with $S_{\text {avg }}^{2}$ values of 0.64 in the free form of the protein and 0.85 in the ternary complex KIX.MLL·pKID and intermediate levels of dynamics for binary complexes KIX.MLL and KIX.pKID $\left(S_{\text {avg }}^{2}\right.$ of 0.80 and 0.71 , respectively). Contrary to the stepwise changes of the order parameters in the L12 loop, which forms direct interactions with MLL in binary KIX.MLL and ternary KIX.MLL·pKID, the three-helix scaffold of the binary KIX complexes rigidifies upon binding a single ligand (either MLL or pKID) and shows no further rigidification upon binding an additional ligand molecule to form the ternary complex KIX·MLL·pKID. This behavior is most prominent for helices $\alpha 1$ and $\alpha 2$ of the KIX domain, helix $\alpha 1$ (KIX $S_{\text {avg }}^{2}=0.84$; KIX.MLL $S_{\text {avg }}^{2}=0.90$; KIX.pKID $S_{\text {avg }}^{2}=0.90$; KIX.MLL·pKID, $\left.S_{\text {avg }}^{2}=0.91\right)$ and $\alpha 2\left(\right.$ KIX $S_{\text {avg }}^{2}=0.82$; KIX.MLL $S_{\text {avg }}^{2}=0.90$; KIX·pKID, $S_{\text {avg }}^{2}=0.91$; KIX.MLL·pKID $\left.S_{\text {avg }}{ }^{2}=0.91\right)$. For helix $\alpha 3$, resonance overlap in unliganded KIX prevents the 
measurement of order parameters for four C-terminal residues of helix $\alpha 3$ (666-669). Comparison of the order parameters of backbone amides in the $\mathrm{N}$-terminal part of helix $\alpha 3$ (646-665), however, suggests that this part of the three-helix scaffold is less dynamic on a pico- to nanosecond time scale in the unliganded protein and only slightly changes its dynamic properties when ligand(s) are bound to the protein, helix $\alpha 3$ (KIX $S_{\text {avg }}^{2}=0.90$; KIX.MLL $S_{\text {avg }}^{2}=0.92$; KIX.pKID $S_{\text {avg }}^{2}=0.91$; KIX.MLL·pKID $S_{\text {avg }}^{2}=0.93$ ).

The KIX backbone amide order parameter data display a relatively large variation for $\alpha 2$ helix in the unliganded form of the protein. An oscillatory behavior of the order parameters is observed for the segment of helix $\alpha 2$ between residues 625637 with lowest order parameters found for residues M625, L628, V629, K632, K633, and E636 and higher order parameters for residues between these. Of note, the more dynamic backbone amides in helix $\alpha 2$ of unliganded KIX are located on the side of the helix that contacts helix $\alpha 1$. We have previously shown that helix $\alpha 1$ is conformationally less stable than helices $\alpha 2$ and $\alpha 3$, with a tendency to unfold and refold (in milliseconds) in the unliganded form of the protein, ${ }^{47}$ suggesting a dynamic coupling of microsecond to millisecond and picosecond to nanosecond motions. The local unfoldingrefolding process is suppressed if MLL or pKID is bound to the KIX domain. ${ }^{17}$ Likewise, upon binding ligand (MLL, pKID, or both), the oscillatory behavior of the order parameters in helix $\alpha 2$ is lost, and helix $\alpha 2$ displays relatively uniform order parameters in ligand bound states.

Taken together, the average order parameters of residues in helical segments of the KIX domain $(\alpha 1-\alpha 3)$ increase from 0.85 in free KIX to 0.91 and 0.90 in the binary complexes KIX.MLL and KIX.pKID, respectively, suggesting significant rigidification of the KIX backbone upon peptide binding. Unlike for the loop L12, formation of the ternary complex KIX.MLL·pKID (average order parameter in $\alpha 1-\alpha 3, S_{\text {avg }}^{2}=$ 0.91 ) is not accompanied by further rigidification of the threehelix bundle scaffold.

\section{DISCUSSION}

NMR relaxation dispersion experiments suggest that allosteric coupling in the KIX domain involves conformational selection of a ca. $7 \%$ low-populated (excited-state) conformer in the binary complex formed by KIX with MLL whose structure resembles the ternary complex. ${ }^{17}$ In agreement with these experimental data, molecular dynamics studies showed that MLL binding to the KIX domain results in a redistribution of the conformational substates of this protein toward conformers that favor the binding of the second, remote ligand. ${ }^{21}$ Side chain methyl group dynamic NMR data indicate that allosteric communication between the MLL and $\mathrm{pKID} / \mathrm{c}-\mathrm{Myb}$ binding sites proceeds through a conserved hydrophobic network in KIX that is formed by the side chains of aliphatic residues. A comparison of binary KIX.MLL and ternary KIX.MLL.pKID complexes of KIX is shown in Figure 1. It is evident that conformational changes of the KIX backbone upon binding of pKID to the binary KIX.MLL complex are indeed minor, which is in agreement with the small magnitude of backbone amide ${ }^{15} \mathrm{~N}$ and backbone carbon ${ }^{13} \mathrm{C}^{\alpha}$ chemical shift changes that are observed upon ligand binding. ${ }^{17}$ Our NMR solution structures show, however, that binding of the pKID activation domain to binary KIX.MLL is accompanied by a small but significant repacking of the hydrophobic core of the KIX domain, involving residues L607, L628, and I660 (Figure 2).
Concomitantly, the side chain of residue I657 flips around its $\chi 1$ dihedral angle from gauche- to gauche+, positioning its $\delta 1$ methyl group at the surface of the KIX domain in the ternary complex. In the ternary complex of KIX with MLL and pKID, the I657 side chain is part of the hydrophobic groove on the surface of KIX that represents the docking interface for pKID, where it hydrophobically packs to residues I137 and L141 of the pKID amphipathic helix $\alpha \mathrm{B}$ (see below). Both I137 and L141 are part of the consensus motif for ligands that bind to the KIX domain. ${ }^{48}$

Allosteric Communication through Redistribution of Rotameric States. It was recently shown that the carbon ${ }^{13} \mathrm{C}$ chemical shifts of methyl groups in isoleucine, leucine, and valine provide a very sensitive measure of the side chain conformations of these residues. ${ }^{49}$ Assuming that conformational sampling of side chain dihedral angles $(\chi 2$ in leucine and isoleucine, $\chi 1$ in valine) can be described in terms of jumps between a limited number of rotameric states, the methyl group ${ }^{13} \mathrm{C}$ chemical shifts of these residues can be used to determine the populations of these rotameric states. For example, with respect to leucine side chain $\chi 2$ dihedral angles, to a very good approximation only the trans and gauche+ conformers are sampled in solution, and their populations can be determined from the difference of their $\delta$-methyl ${ }^{13} \mathrm{C}$ chemical shifts. ${ }^{43}$ Similar approaches are available for isoleucine $\chi^{2}$ and valine $\chi 1$ rotameric distributions. ${ }^{44,45}$

The NMR solution structures of the binary and ternary complexes of the KIX domain that are presented here are based on backbone chemical shifts, NOEs, and residual dipolar couplings as experimental restraints, while isoleucine, leucine, and valine methyl group chemical shifts were not used as restraints in the structure calculations. It is therefore possible to validate the structures by comparison with the rotameric states of isoleucine, leucine, and valine that can be derived from methyl ${ }^{13} \mathrm{C}$ chemical shifts (Table 2). We find excellent agreement between the methyl ${ }^{13} \mathrm{C}$ chemical shifts and the side chain rotamers that are present in the solution structures of both complexes (binary KIX.MLL and ternary KIX.MLL.pKID), suggesting the conformation that is present in the NMR structural bundles represents the most populated rotameric state of isoleucine, leucine, and valine residues in all cases.

It is clear from the chemical shift data, however, that the three-dimensional solution structures of KIX complexes do not fully capture the dynamic nature of this protein. As evident from Table 2, populations of alternative rotameric states of $30 \%$ and more are not uncommon, which is in agreement with other proteins. ${ }^{43,44}$ Intriguingly, however, the chemical shift data suggest that the distributions of isoleucine and leucine $\chi^{2}$ dihedral angles are not identical in binary KIX.MLL and ternary KIX.MLL·pKID: for a subset of residues (L603, L607, I611, L628, L653, I657, and I660) conformational sampling is different in the two complexes, indicating a redistribution of their individual $\chi 2$ rotamers by $4-14 \%$ upon binding of the pKID ligand peptide, as shown in Figure 4. These residues cluster together in the molecular structure of the KIX domain and form part of the hydrophobic core of the protein. This is contrasted by residues L599, L620, L652, L664, L672, and all six valines, for which rotamer populations are identical $(\leq 1 \%$ change) in the two structures and which are located outside the hydrophobic core of KIX. The ${ }^{13} \mathrm{C}$ chemical shift data thus suggest that the conformational transition between the two complexes is accomplished through a relatively subtle $(<14 \%)$ 


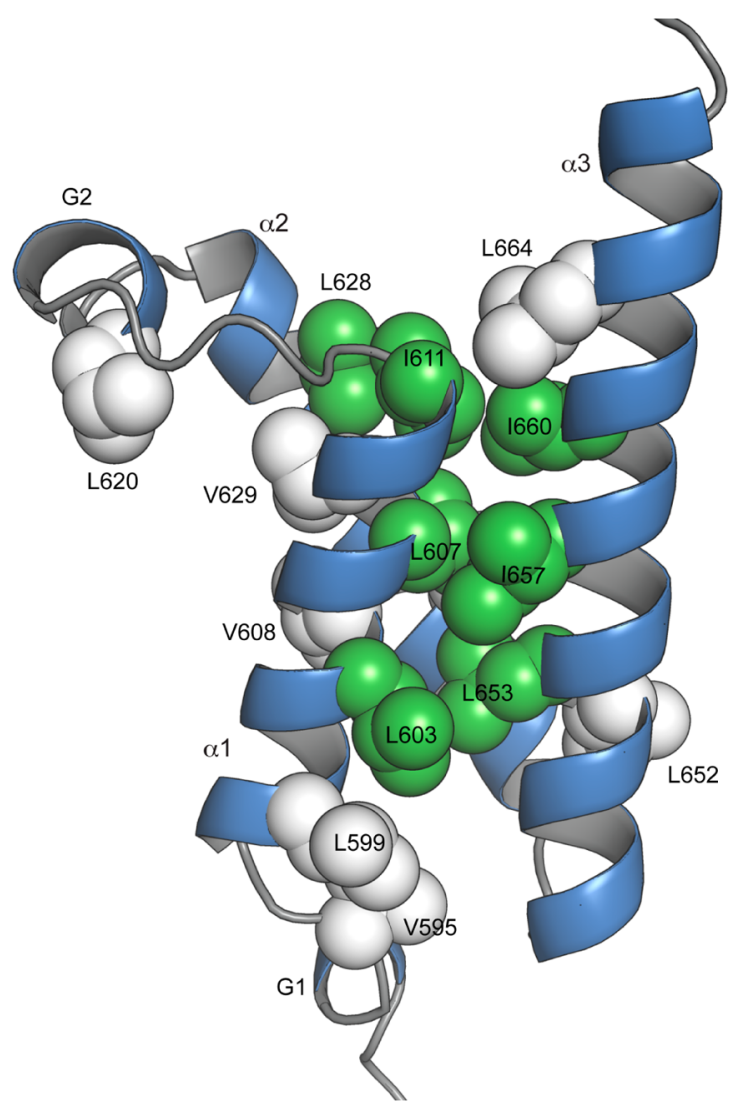

Figure 4. Conformational sampling of side chain dihedral angles $\left(\chi_{2}\right.$ in leucine and isoleucine, $\chi 1$ in valine) in binary KIX.MLL and ternary KIX.MLL·pKID. The backbone of KIX.MLL is shown as ribbon. Leucine, isoleucine, and valine side chains are displayed as spheres and color-coded in green (change of rotamer distribution $\geq 4 \%$ between KIX.MLL and KIX.MLL.pKID) and white (change of rotamer distribution $<2 \%)$.

redistribution of the rotameric states of residues that form the hydrophobic core of the protein.

It is intriguing that the hydrophobic core residues for which we observe the repacking upon ligand binding include the ones that were previously identified to be part of the hydrophobic cluster that constitutes the allosteric network in KIX. ${ }^{15,17}$ Using side chain methyl relaxation dispersion experiments, we found a clear correspondence between the hydrophobic core chemical shifts of the ternary KIX complex and the shifts of the excited state in the binary complex of KIX with MLL. ${ }^{17}$ This shows that the dynamic repacking process of the hydrophobic core that we observe already occurs in binary KIX.MLL (i.e., before pKID binds) during the transition between the 93\% populated ground and the $7 \%$ populated excited states. Taken together, the chemical shift and the structural data provide a quantitative dynamic picture of allosteric communication between the two remote ligand binding sites in the KIX domain.

Figure 5 shows a close-up view of the KIX/pKID binding surface in the ternary complex KIX·MLL·pKID. The $\delta 1$ methyl group of KIX residue I657 is exposed to the surface of the domain and forms part of the hydrophobic pocket that accommodates the amphipathic helix $\alpha \mathrm{B}$ of the ligand peptide pKID. ${ }^{39}$ The hydrophobic side chains of two residues of pKID, I137 and L141, pack to the I657 of the KIX domain. I137 and L141 of pKID are known to be critical for the molecular recognition of ligands binding to the KIX domain: both I137

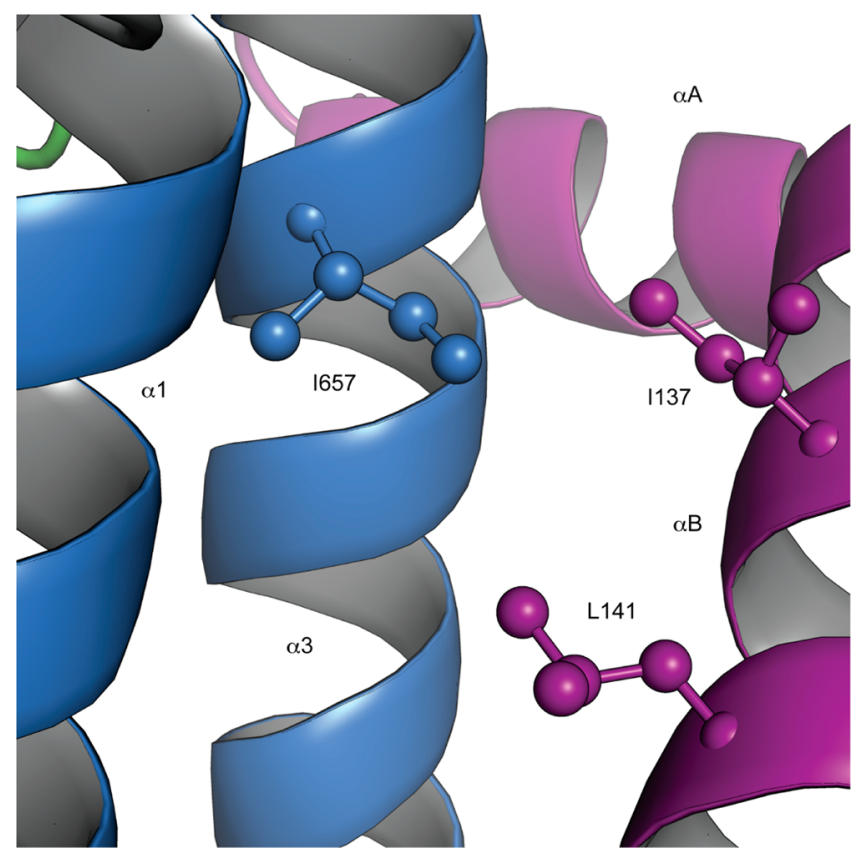

Figure 5. Close-up view of the solution structure of the ternary complex KIX·MLL·pKID showing the interaction surface of the KIX domain with the pKID peptide. The backbone of the KIX domain is displayed as blue ribbon, along with the side chain heavy atoms of I657. The pKID backbone (magenta ribbon) and the side chains of pKID residues I137 and L141, which interact with the hydrophobic side chain of 1657 , are displayed.

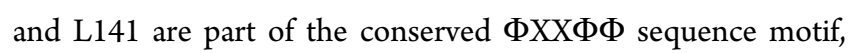
where $\Phi$ is a hydrophobic residue and $\mathrm{X}$ is an arbitrary residue, which is characteristic for ligands that bind to KIX. ${ }^{48}$ Any conformational change at the $\mathrm{pKID} / \mathrm{c}-\mathrm{Myb}$ hydrophobic binding groove will thus very likely modulate the efficiency with which hydrophobic contacts with ligand peptides are formed and affect their affinities. With respect to allosteric coupling, the presence of a higher affinity (excited state) conformer, even if populated only to a low extent, can act as a driving force for the ligand binding process. Recognition and binding of peptides will predominantly occur through the higher affinity conformer, whose structure is complementary to the ligand, while the thermodynamic equilibrium between ground and excited states will be re-established through the allosteric conformational transition, resulting in a redistribution of the conformational substates toward the structure of the higher affinity state. The relaxation dispersion data suggest that in the $7 \%$ populated excited state in the binary complex KIX.MLL residue I657 is indeed present in a conformation that is similar to the ternary complex. ${ }^{17}$

Previous reports have indeed shown that the complementarity of the hydrophobic interactions between the pKID binding pocket and peptide ligands plays a critical role for the affinity with which these ligands are bound to the KIX domain. $^{40}$ NMR solution structures of KIX bound to the activation domain of the transcription factor c-Myb indicate that the positions that are equivalent to I137 and L141 in pKID are taken by two leucine residues in c-Myb (L298 and L302, both part of the c-Myb ФХХФФ motif). It is evident from the KIX.c-Myb structures that the side chain of L302 of c-Myb penetrates more deeply into the hydrophobic pocket, which is formed by the KIX domain, than the equivalent L141 of pKID, ${ }^{39}$ enabling the formation of hydrophobic contacts of its 
methyl groups with the side chain of KIX residue L607 at the bottom of the pocket. Based on these observations, it was suggested that the hydrophobic interactions between pKID and KIX are less optimal than those between pKID and c-Myb, which explains the relatively low affinity of unphosphorylated KID to KIX. ${ }^{40}$

A crystallographic study of KIX bound to a covalently linked small-molecule ligand underlines the remarkable ability of this protein to adapt its hydrophobic core in response to a binding partner. ${ }^{50}$ In this structure, an aromatic ring that is part of the ligand molecule penetrates into the hydrophobic core of the KIX domain, forming contacts with residues that are part of the allosteric network (I611, L607, L628). Binding assays showed that in this small-molecule complex of KIX allosteric communication is disrupted, most likely by engaging the residues that constitute the allosteric network of the protein. Indeed, in the crystal structure of this complex, the side chain $\chi 1$ torsion angle of isoleucine 657 occupies the gaucheconformation, similar to the lower affinity complex KIX.MLL. It thus appears that constraining the hydrophobic core by insertion of a ligand molecule attenuates the malleability of the allosteric network that is required for communication between the binding sites, in agreement with our proposed dynamic allosteric mechanism.

Allosteric Communication in the Opposite Direction. While our relaxation dispersion experiments in combination with the high-resolution structures presented here provide insights into the mechanism by which KIX propagates information about the presence of MLL to the $c-M y b / p K I D$ binding site, the molecular mechanism of communication in the opposite direction (from the c-Myb/pKID binding site to the MLL binding site) is not obvious. ${ }^{17}$ The lack of significant contributions of micro- to millisecond time scale dynamic processes to NMR spin relaxation rates indicates that either the population of any higher energy state(s) that might be present in KIX.pKID or KIX.c-Myb binary complexes is too low or the time scale of the process is outside the micro- to millisecond window that can be studied by relaxation dispersion NMR.

Indeed, the NMR spin relaxation data reported here argue for a contribution of pico- to nanosecond time scale dynamic processes to the mechanism of allosteric coupling from the $\mathrm{c}$ $\mathrm{Myb} / \mathrm{pKID}$ binding site to the MLL binding site. Upon binding of pKID to the KIX domain, we observed a change in pico- to nanosecond dynamics for the KIX backbone for segments of the protein that are remote from the binding site of the peptide. In particular, two of the three helices $(\alpha 1$ and $\alpha 2)$ of the KIX domain that form the three-helix scaffold of the protein rigidify, along with the connecting linker loop L12 and the N-terminus of the domain. These findings are in agreement with the ITC data published by Goto et al., ${ }^{15,17}$ who showed that binding of pKID to KIX decreases the entropic penalty for MLL binding. Furthermore, molecular dynamics studies suggest that $\mathrm{c}-\mathrm{Myb}$ binding to the c-Myb/pKID binding site of KIX indeed rigidifies the loop L12 and G2 region. ${ }^{21}$ This is contrasted by the pKID binding surface itself, which is mostly formed by residues in helix $\alpha 3$ and which does not display a significant change in pico- to nanosecond time scale dynamics upon binding. The rigidification of the linker L12, which has no direct contacts with pKID, is particularly interesting, because it is believed to play a critical role for binding MLL. ${ }^{10}$ Based on the observation of chemical shift averaging and narrow backbone amide resonances, it has been suggested that the linker L12 is conformationally flexible in order to enable the interaction of this binding surface of the KIX domain with a variety of disparate ligand sequences, while in complex with MLL, the conformational flexibility is reduced. ${ }^{10,39}$ In addition, this part of the protein is considerably displaced upon MLL binding, allowing the side chain of KIX residue F612 to form hydrophobic contacts with the MLL ligand. In light of the significance of this loop domain for binding $M L L,{ }^{21}$ this longrange dynamic coupling between the two binding sites is likely of functional significance.

A markedly different behavior is found for MLL binding to KIX. While the presence of bound MLL significantly increases the order parameters of amino acid residues that are part of the hydrophobic MLL binding pocket (loop L12) and the helices $\alpha 1$ and $\alpha 2$ of the three-helix scaffold of the protein as well as the C-terminal end of helix $\alpha 3$, we find no significant changes of order parameters at the pKID binding site. Thus, binding of the activation domain of MLL appears to cause local rigidification but has no long-range effect on dynamic processes on the pico- to nanosecond time scale. Interestingly, however, the loss of flexibility on the pico- to nanosecond time scale upon MLL binding of the L12 loop is accompanied by a significant increase of dynamics on a millisecond time scale (the allosteric conformational transition) that involves a contiguous network of residues connecting the L12 loop to the remote pKID binding site of the KIX domain. ${ }^{17}$

Dynamics and Allostery. Dynamic allosteric coupling has been reported for a variety of proteins. ${ }^{51-55}$ For example, various variants of the catabolite activator protein (CAP) differentially populate active and inactive DNA-binding states. ${ }^{56}$ Allosteric regulation of CAP is entirely of an entropic nature and involves the redistribution of the conformational substates of the protein in response to the presence of a ligand molecule. ${ }^{57}$ Likewise, phosphorylation of the bacterial nitrogen regulatory protein $\mathrm{C}$ shifts the balance of populations of the different forms of this protein from the inactive to the active form, ${ }^{58}$ and the PBX1 homeodomain transiently folds into a conformation in which the binding sites for DNA and the transcription factor Hox are preorganized even in the absence of ligands. ${ }^{59}$ In PDZ3, binding function is regulated through an entirely dynamic allosteric mechanism that lacks measurable conformational changes. ${ }^{60}$ Together with our results for the KIX domain of CBP, these data underscore the mechanistic significance of dynamic equilibria for allosteric regulation and ligand recognition. Of note, such population shift mechanisms enable the modulation of binding affinities in a versatile manner by the extent to which the higher affinity conformer is populated, as well as by the difference in binding affinities of the different conformers.

It is noteworthy that the KIX domain displays a nonuniform distribution of aromatic and aliphatic residues in its hydrophobic core. The interior of the hydrophobic core (within the three-helix scaffold) is formed by nonaromatic residues and contains mostly isoleucines, leucines, valines, and alanines, while at the surface of KIX, aromatic residues are enriched, making van der Waals contacts with the bound ligand peptides. $^{10,39,41}$ A possible involvement of these aromatic residues at the protein-ligand interface in allosteric coupling has been inferred from relaxation dispersion and ${ }^{19} \mathrm{~F}$ NMR measurements, ${ }^{17,61}$ but their exact mechanistic role is not evident. Considering the dynamic nature of the repacking process of isoleucine, leucine, and valine side chains in the KIX hydrophobic core, it is tempting to speculate that aliphatic side chains could be more amenable for such processes than 
aromatic side chains. Sequence comparison showed that the aliphatic residues in the KIX hydrophobic core are conserved to a higher level than the aromatic residues, ${ }^{17}$ arguing for an evolutionary conservation of such functional dynamics. ${ }^{62,63}$ However, further experimental studies of allosteric proteins, including techniques that probe the dynamic behavior of aromatic side chains, will be necessary to comprehensively understand the structural prerequisites for dynamic allosteric coupling.

\section{ASSOCIATED CONTENT}

\section{Accession Codes}

Structural coordinates and NMR restraints have been deposited in the Protein Data Bank and BioMagResBank with the following accession numbers: KIX.MLL (PDB ID 2LXS; BMRB 18694) and KIX.MLL·pKID (PDB ID 2LXT; BMRB 18695).

\section{AUTHOR INFORMATION}

\section{Corresponding Author}

*E-mail: martin.tollinger@uibk.ac.at.

\section{Present Address}

${ }^{\S}$ S. Brüschweiler: Department of Biological Chemistry and Molecular Pharmacology, Harvard Medical School, Boston, Massachusetts 02115, USA.

\section{Notes}

The authors declare no competing financial interest.

\section{ACKNOWLEDGMENTS}

We are grateful to D. Flemming Hansen (University College London) for insightful discussions. We thank Paul Schanda (IBS Grenoble, France) for preliminary alignment experiments. This work was supported by the Austrian Science Fund FWF (P22735).

\section{REFERENCES}

(1) Carey, M. (1998) The enhanceosome and transcriptional synergy. Cell 92, 5-8.

(2) Panne, D. (2008) The enhanceosome. Curr. Opin. Struct. Biol. 18, $236-242$.

(3) Ptashne, M., and Gann, A. (1997) Transcriptional activation by recruitment. Nature 386, 569-577.

(4) Lemon, B., and Tjian, R. (2000) Orchestrated response: A symphony of transcription factors for gene control. Genes Dev. 14, 2551-2569.

(5) Goodman, R. H., and Smolik, S. (2000) CBP/p300 in cell growth, transformation, and development. Genes Dev. 14, 1553-1577.

(6) Kornberg, R. D. (2005) Mediator and the mechanism of transcriptional activation. Trends. Biochem. Sci. 30, 235-239.

(7) Merika, M., and Thanos, D. (2001) Enhanceosomes. Curr. Opin. Genet. Dev. 11, 205-208.

(8) Vo, N., and Goodman, R. H. (2001) CREB-binding protein and p300 in transcriptional regulation. J. Biol. Chem. 276, 13505-13508.

(9) Dyson, H. J., and Wright, P. E. (2005) Intrinsically unstructured proteins and their functions. Nat. Rev. Mol. Cell. Biol. 6, 197-208.

(10) De Guzman, R. N., Goto, N. K., Dyson, H. J., and Wright, P. E. (2006) Structural basis for cooperative transcription factor binding to the CBP coactivator. J. Mol. Biol. 355, 1005-1013.

(11) Yang, F., Vought, B. W., Satterlee, J. S., Walker, A. K., Jim Sun, Z. Y., Watts, J. L., DeBeaumont, R., Saito, R. M., Hyberts, S. G., Yang, S., Macol, C., Iyer, L., Tjian, R., van den Heuvel, S., Hart, A. C., Wagner, G., and Naar, A. M. (2006) An ARC/Mediator subunit required for SREBP control of cholesterol and lipid homeostasis. Nature 442, 700-704.
(12) Thakur, J. K., Arthanari, H., Yang, F., Pan, S. J., Fan, X., Breger, J., Frueh, D. P., Gulshan, K., Li, D. K., Mylonakis, E., Struhl, K., MoyeRowley, W. S., Cormack, B. P., Wagner, G., and Naar, A. M. (2008) A nuclear receptor-like pathway regulating multidrug resistance in fungi. Nature 452, 604-609.

(13) Ernst, P., Wang, J., Huang, M., Goodman, R. H., and Korsmeyer, S. J. (2001) MLL and CREB bind cooperatively to the nuclear coactivator CREB-binding protein. Mol. Cell. Biol. 21, 2249-2258.

(14) Wang, F., Marshall, C. B., Yamamoto, K., Li, G. Y., GasmiSeabrook, G. M., Okada, H., Mak, T. W., and Ikura, M. (2012) Structures of KIX domain of CBP in complex with two FOXO3a transactivation domains reveal promiscuity and plasticity in coactivator recruitment. Proc. Natl. Acad. Sci. U.S.A. 109, 6078-6083.

(15) Goto, N. K., Zor, T., Martinez-Yamout, M., Dyson, H. J., and Wright, P. E. (2002) Cooperativity in transcription factor binding to the coactivator CREB-binding protein (CBP). J. Biol. Chem. 277, 43168-43174.

(16) Cheng, X., Reginato, M. J., Andrews, N. C., and Lazar, M. A. (1997) The transcriptional integrator CREB-binding protein mediates positive cross talk between nuclear hormone receptors and the hematopoietic bZip protein p45/NF-E2. Mol. Cell. Biol. 17, 14071416.

(17) Brüschweiler, S., Schanda, P., Kloiber, K., Brutscher, B., Kontaxis, G., Konrat, R, and Tollinger, M. (2009) Direct observation of the dynamic process underlying allosteric signal transmission. J. Am. Chem. Soc. 131, 3063-3068.

(18) Tsai, C. J., Kumar, S., Ma, B., and Nussinov, R. (1999) Folding funnels, binding funnels, and protein function. Protein Sci. 8, 11811190.

(19) Weber, G. (1972) Ligand binding and internal equilibria in proteins. Biochemistry 11, 864-878.

(20) Tobi, D., and Bahar, I. (2005) Structural changes involved in protein binding correlate with intrinsic motions of proteins in the unbound state. Proc. Natl. Acad. Sci. U.S.A. 102, 18908-18913.

(21) Korkmaz, E. N., Nussinov, R., and Haliloglu, T. (2012) Conformational control of the binding of the transactivation domain of the MLL protein and c-Myb to the KIX domain of CREB. PLoS Comput. Biol. 8, No. e1002420.

(22) Tollinger, M., Kloiber, K., Agoston, B., Dorigoni, C., Lichtenecker, R., Schmid, W., and Konrat, R. (2006) An isolated helix persists in a sparsely populated form of KIX under native conditions. Biochemistry 45, 8885-8893.

(23) Delaglio, F., Grzesiek, S., Vuister, G. W., Zhu, G., Pfeifer, J., and Bax, A. (1995) NMRPipe: A multidimensional spectral processing system based on UNIX pipes. J. Biomol. NMR 6, 277-293.

(24) Vranken, W. F., Boucher, W., Stevens, T. J., Fogh, R. H., Pajon, A., Llinas, M., Ulrich, E. L., Markley, J. L., Ionides, J., and Laue, E. D. (2005) The CCPN data model for NMR spectroscopy: development of a software pipeline. Proteins 59, 687-696.

(25) Neri, D., Szyperski, T., Otting, G., Senn, H., and Wuthrich, K. (1989) Stereospecific nuclear magnetic resonance assignments of the methyl groups of valine and leucine in the DNA-binding domain of the 434 repressor by biosynthetically directed fractional 13C labeling. Biochemistry 28, 7510-7516.

(26) Zwahlen, C., Gardner, K. H., Sarma, S. P., Horita, D. A., Byrd, R A., and Kay, L. E. (1998) An NMR experiment for measuring methylmethyl NOEs in C-13-labeled proteins with high resolution. J. Am. Chem. Soc. 120, 7617-7625.

(27) Eichmüller, C., Schüler, W., Konrat, R., and Kräutler, B. (2001) Simultaneous measurement of intra- and intermolecular NOEs in differentially labeled protein-ligand complexes. J. Biomol. NMR 21, $107-116$.

(28) Chou, J. J., Gaemers, S., Howder, B., Louis, J. M., and Bax, A. (2001) A simple apparatus for generating stretched polyacrylamide gels, yielding uniform alignment of proteins and detergent micelles. $J$. Biomol. NMR 21, 377-382.

(29) Ottiger, M., Delaglio, F., and Bax, A. (1998) Measurement of J and dipolar couplings from simplified two-dimensional NMR spectra. J. Magn. Reson. 131, 373-378. 
(30) Ortega-Roldan, J. L., Jensen, M. R., Brutscher, B., Azuaga, A. I., Blackledge, M., and van Nuland, N. A. (2009) Accurate characterization of weak macromolecular interactions by titration of NMR residual dipolar couplings: application to the CD2AP SH3-C:ubiquitin complex. Nucleic Acids Res. 37, e70.

(31) Brüschweiler, R., Liao, X., and Wright, P. E. (1995) Long-range motional restrictions in a multidomain zinc-finger protein from anisotropic tumbling. Science 268, 886-889.

(32) Lee, L. K., Rance, M., Chazin, W. J., and Palmer, A. G., 3rd. (1997) Rotational diffusion anisotropy of proteins from simultaneous analysis of $15 \mathrm{~N}$ and $13 \mathrm{C}$ alpha nuclear spin relaxation. J. Biomol. NMR 9, 287-298.

(33) Cole, R., and Loria, J. P. (2003) FAST-Modelfree: A program for rapid automated analysis of solution NMR spin-relaxation data. $J$. Biomol. NMR 26, 203-213.

(34) Shen, Y., Delaglio, F., Cornilescu, G., and Bax, A. (2009) TALOS+: A hybrid method for predicting protein backbone torsion angles from NMR chemical shifts. J. Biomol. NMR 44, 213-223.

(35) Rieping, W., Habeck, M., Bardiaux, B., Bernard, A., Malliavin, T. E., and Nilges, M. (2007) ARIA2: Automated NOE assignment and data integration in NMR structure calculation. Bioinformatics 23, 381382.

(36) Zweckstetter, M., Hummer, G., and Bax, A. (2004) Prediction of charge-induced molecular alignment of biomolecules dissolved in dilute liquid-crystalline phases. Biophys. J. 86, 3444-3460.

(37) Brunger, A. T. (2007) Version 1.2 of the crystallography and NMR system. Nat. Protoc. 2, 2728-2733.

(38) Linge, J. P., Williams, M. A., Spronk, C. A., Bonvin, A. M., and Nilges, M. (2003) Refinement of protein structures in explicit solvent. Proteins 50, 496-506.

(39) Radhakrishnan, I., Perez-Alvarado, G. C., Parker, D., Dyson, H. J., Montminy, M. R., and Wright, P. E. (1997) Solution structure of the KIX domain of CBP bound to the transactivation domain of CREB: a model for activator:coactivator interactions. Cell 91, 741-752.

(40) Zor, T., De Guzman, R. N., Dyson, H. J., and Wright, P. E. (2004) Solution structure of the KIX domain of CBP bound to the transactivation domain of c-Myb. J. Mol. Biol. 337, 521-534.

(41) Denis, C. M., Chitayat, S., Plevin, M. J., Wang, F., Thompson, P., Liu, S., Spencer, H. L., Ikura, M., LeBrun, D. P., and Smith, S. P. (2012) Structural basis of $\mathrm{CBP} / \mathrm{p} 300$ recruitment in leukemia induction by E2A-PBX1. Blood 120, 3968-3977.

(42) Arai, M., Dyson, H. J., and Wright, P. E. (2010) Leu628 of the KIX domain of CBP is a key residue for the interaction with the MLL transactivation domain. FEBS Lett. 584, 4500-4504.

(43) Mulder, F. A. (2009) Leucine side-chain conformation and dynamics in proteins from 13C NMR chemical shifts. ChemBioChem $10,1477-1479$.

(44) Hansen, D. F., Neudecker, P., and Kay, L. E. (2010) Determination of isoleucine side-chain conformations in ground and excited states of proteins from chemical shifts. J. Am. Chem. Soc. 132, $7589-7591$.

(45) Hansen, D. F., and Kay, L. E. (2011) Determining valine sidechain rotamer conformations in proteins from methyl $13 \mathrm{C}$ chemical shifts: application to the $360 \mathrm{kDa}$ half-proteasome. J. Am. Chem. Soc. $133,8272-8281$.

(46) Mittermaier, A., and Kay, L. E. (2006) New tools provide new insights in NMR studies of protein dynamics. Science 312, 224-228.

(47) Schanda, P., Brutscher, B., Konrat, R., and Tollinger, M. (2008) Folding of the KIX domain: characterization of the equilibrium analog of a folding intermediate using ${ }^{15} \mathrm{~N} /{ }^{13} \mathrm{C}$ relaxation dispersion and fast ${ }^{1} \mathrm{H} /{ }^{2} \mathrm{H}$ amide exchange NMR spectroscopy. J. Mol. Biol. 380, 726-741.

(48) Plevin, M. J., Mills, M. M., and Ikura, M. (2005) The LxxLL motif: A multifunctional binding sequence in transcriptional regulation. Trends Biochem. Sci. 30, 66-69.

(49) London, R. E., Wingad, B. D., and Mueller, G. A. (2008) Dependence of amino acid side chain $13 \mathrm{C}$ shifts on dihedral angle: application to conformational analysis. J. Am. Chem. Soc. 130, 1109711105 .
(50) Wang, N., Majmudar, C. Y., Pomerantz, W. C., Gagnon, J. K., Sadowsky, J. D., Meagher, J. L., Johnson, T. K., Stuckey, J. A., Brooks, C. L., 3rd, Wells, J. A., and Mapp, A. K. (2013) Ordering a dynamic protein via a small-molecule stabilizer. J. Am. Chem. Soc. 135, 33633366.

(51) Swain, J. F., and Gierasch, L. M. (2006) The changing landscape of protein allostery. Curr. Opin. Struct. Biol. 16, 102-108.

(52) Kern, D., and Zuiderweg, E. R. (2003) The role of dynamics in allosteric regulation. Curr. Opin. Struct. Biol. 13, 748-757.

(53) Goodey, N. M., and Benkovic, S. J. (2008) Allosteric regulation and catalysis emerge via a common route. Nat. Chem. Biol. 4, 474-482.

(54) Csermely, P., Palotai, R., and Nussinov, R. (2010) Induced fit, conformational selection and independent dynamic segments: an extended view of binding events. Trends Biochem. Sci. 35, 539-546.

(55) Cui, Q., and Karplus, M. (2008) Allostery and cooperativity revisited. Protein Sci. 17, 1295-1307.

(56) Tzeng, S. R., and Kalodimos, C. G. (2012) Protein activity regulation by conformational entropy. Nature 488, 236-240.

(57) Kalodimos, C. G. (2012) Protein function and allostery: A dynamic relationship. Ann. N.Y. Acad. Sci. 1260, 81-86.

(58) Volkman, B. F., Lipson, D., Wemmer, D. E., and Kern, D. (2001) Two-state allosteric behavior in a single-domain signaling protein. Science 291, 2429-2433.

(59) Farber, P. J., and Mittermaier, A. (2011) Concerted dynamics link allosteric sites in the PBX homeodomain. J. Mol. Biol. 405, 819830.

(60) Petit, C. M., Zhang, J., Sapienza, P. J., Fuentes, E. J., and Lee, A. L. (2009) Hidden dynamic allostery in a PDZ domain. Proc. Natl. Acad. Sci. U.S.A. 106, 18249-18254.

(61) Pomerantz, W. C., Wang, N., Lipinski, A. K., Wang, R., Cierpicki, T., and Mapp, A. K. (2012) Profiling the dynamic interfaces of fluorinated transcription complexes for ligand discovery and characterization. ACS Chem. Biol. 7, 1345-1350.

(62) Lockless, S. W., and Ranganathan, R. (1999) Evolutionarily conserved pathways of energetic connectivity in protein families. Science 286, 295-299.

(63) Süel, G. M., Lockless, S. W., Wall, M. A., and Ranganathan, R. (2003) Evolutionarily conserved networks of residues mediate allosteric communication in proteins. Nat. Struct. Biol. 10, 59-69.

(64) DeLano, W. L. (2002), San Carlos, Ca, USA. 